\title{
THE R-BAND HUBBLE DIAGRAM FOR GPS GALAXIES.
}

\author{
IGNAS SNELLEN ${ }^{1}$, MALCOLM BREMER ${ }^{1,2}$, RICHARD SCHILIZZI $^{3,1}$, \\ GEORGE MILEY ${ }^{1}$ AND ROB VAN OJIK ${ }^{1}$ \\ 1 Leiden Observatory, The Netherlands \\ 2 Institute of Astronomy, Cambridge, U.K. \\ 3 Joint Institute for VLBI in Europe, Dwingeloo, The Netherlands
}

\begin{abstract}
The Hubble diagram of GPS galaxies has a low dispersion and a steep slope compared with that for $3 \mathrm{C}$ galaxies. The relative faintness of GPS galaxies at high redshift may be due to the absence of the aligned optical/uv component seen in high redshift $3 \mathrm{C}$ galaxies. The GPS Hubble relation is too steep to fit with evolution models for passively evolving ellipticals. This could be caused by the dynamical evolution of the GPS galaxies.
\end{abstract}

Gigahertz Peaked Spectrum (GPS) sources are a class of compact extragalactic radio source with a dominant peak in their radio spectrum at about $1 \mathrm{GHz}$ in frequency. It is believed that GPS sources are confined within the inner regions of their host galaxies, either because they are young radio sources that will evolve into extended radio galaxies (Readhead et al 1994, Fanti et al 1995), or because they are surrounded by a particular dense interstellar medium (O'Dea et al 1991).

We are carrying out a project to investigate the nature of these objects by studying a sample of faint GPS sources from the Westerbork Northern Sky Survey (WENSS), and comparing them with brighter samples (e.g. O'Dea et al 1991, Stanghellini et al 1994).

While making an inventory of the optical properties of bright GPS sources we noted that the Hubble diagram of GPS galaxies has a low dispersion and a steep slope compared with that for $3 \mathrm{C}$ galaxies. A detailed description of this work can be found in Snellen et al 1995.

Figure 1 shows the R-band Hubble diagram for a sample of 22 GPS galaxies found in the literature. Also shown are the Hubble diagram for $3 \mathrm{C}$ 


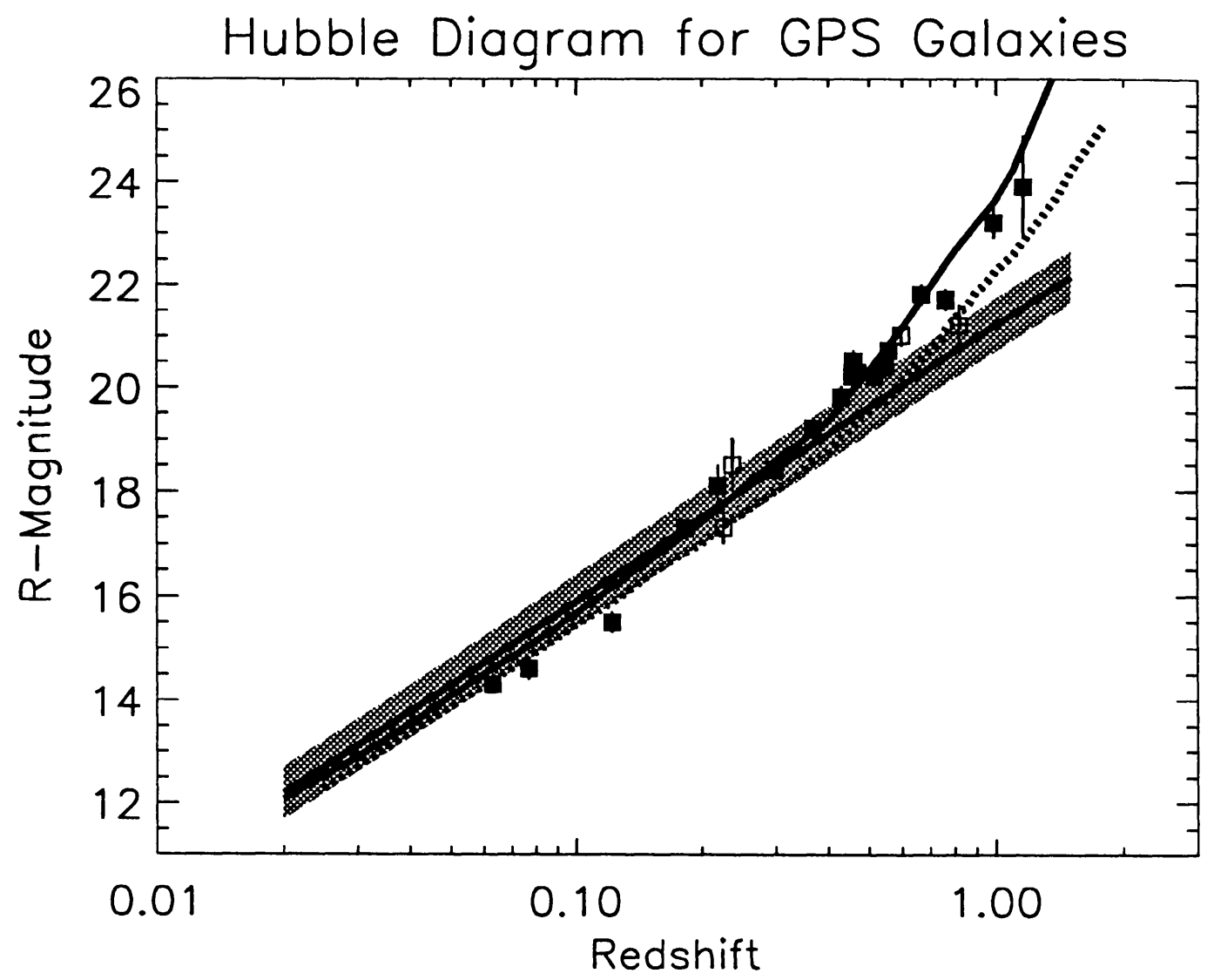

Figure 1. The R-band Hubble diagram for GPS galaxies. The open squares indicate the GPS galaxies at low galactic latitude $\left(b<15^{\circ}\right)$. Note the low dispersion and steep slope of the GPS Hubble relation. The grey band indicates the Hubble relation for $3 \mathrm{C}$ galaxies, The $3 \mathrm{C}$ galaxies are clearly brighter at high redshift. The solid line is the expected curve for non-evolving ellipticals. The dotted line is the expected curve for passively evolving ellipticals (Bruzual, C-model).

galaxies and models for passively evolving and non-evolving ellipticals. The dispersion around a least squares fit is 0.37 magnitudes, but decreases to 0.28 magnitudes when the four galaxies with the lowest galactic latitude are excluded due to the uncertainty in galactic extinction.

The dispersion is low and the slope is steep in comparison with the Hubble diagram for $3 \mathrm{C}$ galaxies (Eales et al 1985). The dispersion and slope are comparable with the Hubble diagram for first ranked cluster galaxies (Hoessel et al 1980), although that is only determined at low redshift. The relation is too steep to fit with a passively evolving elliptical model (Bruzual 1983), but it can be fitted with a model of an elliptical galaxy with an old stellar population which is not evolving (Coleman et al 1980) 


\section{Discussion}

It appears that the Hubble diagram for GPS galaxies is consistent with them having an old stellar population that undergoes no cosmological evolution. That the galaxies are in a special phase of their evolution (the "GPS" phase) could explain this. However, nearby GPS galaxies show a close companion or double nucleus, which indicates that galactic cannibalism (dynamical evolution) may play an important role in these objects. It is possible that the dynamical and stellar evolution cancel each other out more or less, which makes the Hubble diagram consistent with a non-evolution scenario. This means that the mass of a GPS galaxy would need to increase typically by a factor $2-3$ between a redshift of 1 and 0.1 , assuming $M / L$ is constant.

We suggest that the GPS galaxies are fainter at high redshift than $3 \mathrm{C}$ galaxies, because the former do not have the extra component seen in high redshift $3 \mathrm{C}$ galaxies responsible for the optical/radio alignment effect. This aligned light usually completely dominates the rest-frame UV emission of $3 \mathrm{C}$ galaxies at $z>0.6$, although there is wide variation from source to source. If GPS sources are missing this extra aligned component of emission, this would explain why the R-band magnitude of the GPS galaxies are similar to $3 \mathrm{C}$ galaxies at low redshift, but fainter at higher redshifts. This would also explain the larger scatter in the $3 \mathrm{C}$ Hubble relation, due to source-to-source variation in the aligned component.

The comparable magnitudes and dispersions of the Hubble relations for GPS galaxies and first ranked cluster galaxies suggest that GPS galaxies are located in the centres of clusters. However there is, as yet, no observational evidence for this.

\section{References}

Bruzual G.A., 1983, Rev. Mex. Astr. Astrofiz.,8, 63

Coleman G.D., Chi-Chao Wu, Weedman D.W., 1980, Astrophys. J. Suppl., 43, 393

Eales S.A., 1985, Mon. Not. R. Astr. Soc., 213, 899

Fanti C., Fanti R., Dallacasa D., Schilizzi R.T., Spencer R.E., Stanghellini C., Astr. Astrophys., in press

Hoessel J.G., Gunn J.E. and Thuan T.X., 1980, Astrophys. J., 241, 486

O'Dea C.P., Baum S.A., Stanghellini C., 1991, Astrophys. J., 66, 380

Readhead A.C.S., Xu W., Pearson T.J., 1994, in Compact Extragalactic Radio Sources, ed. J.A. Zensus and K.I. Kellermann, p17

Snellen I., Bremer M., Schilizzi R., Miley G., van Ojik R., 1995, Mon. Not. R. Astr. Soc., in press

Stanghellini C., O’Dea C.P., Baum S.A., Laurikanen E., 1993, Astrophys. J. Suppl., 88,1 